Conclusions Waist skeletal muscle volume is a novel prognostic biomarker in patients with endometrial cancer. Assessing body composition before treatment may provide important prognostic information for such patients.

\section{EPV128/\#510 WOMB CANCER RISK AWARENESS: DEVELOPING TOOLS TO INFLUENCE CHANGE}

O Jones* ${ }^{*}$ H Young, H Clarke, EJ Crosbie, VN Sivalingam. University of Manchester, Division of Cancer Sciences, School of Medical Sciences, Manchester, UK

\subsection{6/ijgc-2021-IGCS.198}

Objectives Endometrial or womb cancer is the most common gynaecological malignancy in the developed world. Efficient and cost-effective methods of increasing public awareness about womb cancer are research priorities for patients and clinicians. Until now, there has been no accepted measure of womb cancer awareness. We aimed to develop the self-complete Womb Cancer Awareness Measure(WCAM).

Methods Relevant questions on warning signs, risk factors and existing cancer screening programmes were extracted from the literature and ratified by patients and expert clinicians. Reliability and validity were assessed in female research participants aged $19-65(n=65)$ and expert clinicians $(n=10)$. Readability was calculated using the Flesch Reading Ease formula. Test-retest reliability was tested over two weeks. Construct reliability was established by comparing scores of expert clinicians and non-medical academics. Sensitivity to change was measured by comparing participants who read a womb cancer leaflet against a control leaflet.

Results The readability of the WCAM was high(71\%). Testretest reliability revealed high percentage exact agreement of $78-80 \%$ for all items. Discrepancies were due to improvement in the second score, demonstrating that the WCAM completion increased knowledge and awareness. Experts achieved higher knowledge scores than non-medical academics indicating good construct validity $(\mathrm{p}<0.001)$. The measure was sensitive to change; the womb cancer leaflet group $(n=22)$ scored higher for cancer awareness [mean 70(13)] than the controls $(\mathrm{n}=21)[$ mean $54(6.2)](\mathrm{p}<0.001)$.

Conclusions This study demonstrates the psychometric validity of the WCAM and its potential for use for further testing. Ongoing work will extensively validate this awareness measure in an ethnically and socioeconomically diverse population including women at increased risk of womb cancer.

\section{EPV129/\#529 LONG-TERM CLINICAL AND ECONOMIC VALUE OF PEMBROLIZUMAB + LENVATINIB COMPARED WITH CHEMOTHERAPY IN PREVIOUSLY TREATED ADVANCED ENDOMETRIAL CANCER PATIENTS IN SWEDEN: A COST-EFFECTIVENESS ANALYSIS}

${ }^{1} \mathrm{~N}$ Upadhyay ${ }^{*},{ }^{2} \mathrm{~L}$ Ralph, ${ }^{3} \mathrm{C}$ Ljungcrantz, ${ }^{2} \mathrm{~A}$ Merchant, ${ }^{2} \mathrm{~B}$ Haycroft, ${ }^{4} \mathrm{~A}$ Fornwall, ${ }^{1} V$ Prabhu, ${ }^{5} \mathrm{R}$ Orlowski, ${ }^{6} \mathrm{~L}$ Duska. ${ }^{1}$ Merck and Co., Inc., Center of Observational and Real World Evidence, Kenilworth, USA; ${ }^{2}$ BresMed Health Solutions Ltd, Health Economics, Sheffield, UK; ${ }^{3}$ MSD Sweden, Market Access, Stockholm, Sweden; ${ }^{4}$ Quantify Research, Health Economics, Stockholm, Sweden; ${ }^{5}$ Merck and Co., Inc., Late Stage Clinical Development, Kenilworth, USA; ${ }^{6}$ University of Virginia, Department of Obstetrics and Gynecology, Charlottesville, USA

10.1136/ijgc-2021-IGCS.199
Objectives Advanced endometrial cancer (aEC) patients previously treated with systemic therapy have limited treatment options in Europe. In the Phase-III trial KEYNOTE-775, pembrolizumab + lenvatinib (PEM+LEN) demonstrated statistically significant and clinically meaningful improvements in OS, PFS and ORR versus chemotherapy (the treatment of physician's choice [TPC] of doxorubicin or paclitaxel). The long-term clinical and economic value of PEM+LEN needs to be understood. The objective of this study was to assess the cost-effectiveness of PEM+LEN vs TPC for previously treated aEC patients in Sweden.

Methods A three-state partitioned survival model (progression free, progressed disease, and death) was developed. The proportion of patients in each health state was estimated using the area under the curve based on $\mathrm{KN}-775$ OS and PFS data, to which costs/benefits from a Swedish healthcare perspective were applied over a lifetime horizon. OS, PFS, time-on-treatment, adverse event, and EQ-5D utility data were obtained from KEYNOTE-775. Treatment acquisition, administration, resource use and adverse events cost were obtained from Sweden. A 3\% discount rate was applied. Sensitivity analyses were conducted.

Results Treatment with PEM+LEN resulted in an increase of 1.96 Life-years (LYs), 1.42 quality-adjusted life-years (QALYs), and SEK 1,180,044 in costs vs chemotherapy (TPC). The incremental cost-effectiveness ratio for PEM+LEN vs chemotherapy was 828,569 SEK/QALY-gained. Cost-effectiveness results were sensitive to OS/time-on-treatment extrapolations, and adjustments for subsequent therapies.

Conclusions Model-based analysis suggests that PEM+LEN extends life-years and QALYs over chemotherapy, and can be considered cost-effective compared with chemotherapy at a willingness-to-pay threshold of SEK 1-million in Sweden.

\section{EPV130/\#537 ENDOMETRIAL CANCER (EC): LYMPHOVASCULAR SPACE INVASION (LVSI) AND LYMPH NODE METASTASIS (LNM) ACCORDING TO MOLECULAR SUBGROUPS}

${ }^{1} \mathrm{C}$ Del Pezzo* ${ }^{*} \mathrm{D}$ Scaldaferri, ${ }^{2} \mathrm{M}$ Giordano, ${ }^{1} \mathrm{E}$ Fuggetta, ${ }^{1} \mathrm{C}$ De Marco, ${ }^{1} \mathrm{~F}$ Maneschi. ${ }^{1}$ Azienda Ospedaliera San Giovanni Addolorata, Gyn/obs Unit, Roma, Italy; ${ }^{2}$ Azienda Ospedaliera San Giovanni Addolorata, Pathology Unit, Roma, Italy

\subsection{6/ijgc-2021-IGCS.200}

Objectives To evaluate the distribution of LVSI and LNM according to the EC molecular classification.

Methods Patients with EC surgically treated were retrospectively analyzed. Tumor grade and histologic subtype were assessed by HE technique. MMR and p53 status were assessed by IHC in all patients. POLE was sequentiated in 6 LCN G3 patients. Chi-square test was adopted for categorical data. Odds-ratio was adopted to evaluate association.

Results 70 consecutive patients entered the study: endometrioid type was found in 61 (87.1\%); G1-2 in $44(62.9 \%)$ and G3 in $26(37.1 \%)$ patients, respectively. Molecular profiling classified $3(4.3 \%)$ as POLE-ultramutated, 34 (48.6\%) as LCN, 22 tumors (31.4\%) as MMRd and 11 $(15.7 \%)$ as p53-mutated. LVSI was found in 18 (25.7\%) patients: $0 / 3 \quad(0 \%) \quad$ u-POLE, 6/34 (17.6\%) $\quad$ LCN, 6/22 (27.3\%) MMRd and 6/11 (54.5\%) p53-mutated ( $\mathrm{p}=0,07)$. LNM were present in $17(24.3 \%)$ cases: 0/3 (0\%) u-POLE, $5 / 34(14.7 \%)$ LCN, 5/22 (22.7\%) MMRd and 7/11 (63.6\%) 
p53-mutated ( $\mathrm{p}<0,01)$. MMRd vs LCN: OR 1.75 (95\% CI $0.48-6.34, \mathrm{p}=0.39$ ) for LVSI and 1.70 (95\% CI $0.43-6.75$, $\mathrm{p}=0.44)$ for LNM.

Conclusions The rate of LVSI and LNM showed a significantly increasing trend from u-POLE to p53-mutated. Among the MMRd and LCN molecular subtypes with intermediate prognostic impact, where the classical prognostic parameter may have a role, MMRd patients had a higher, although not significant, risk of LVSI and LNM. The reduced number of patients, which is one of the limit of the study may explain the lack of significance. Larger studies are suggested.

\section{EPV131/\#545 LOWER UTERINE SEGMENT INVOLVEMENT IN HIGH-GRADE ENDOMETRIAL CARCINOMA IS NOT INDEPENDENTLY ASSOCIATED WITH ADVERSE ONCOLOGICAL OUTCOME}

${ }^{1} \mathrm{~B}$ Brandt, ${ }^{1} \mathrm{~T}$ Perri, ${ }^{2} \mathrm{~L}$ Helpman, ${ }^{3} \mathrm{R}$ Eitan, ${ }^{4} \mathrm{Z}$ Vaknin, ${ }^{5} \mathrm{O}$ Lavie, ${ }^{6} \mathrm{~A}$ Ben Arie, ${ }^{7} \mathrm{~A}$ Amit, ${ }^{8} \mathrm{~T}$ Levy, ${ }^{9} \mathrm{~A}$ Namazov, ${ }^{10}$ | Ben Shachar, ${ }^{11}$ | Atlas, ${ }^{12}$ | Bruchim, ${ }^{1} \mathrm{G}$ Levin ${ }^{*},{ }^{13} \mathrm{O}$ Gemer. ${ }^{1}$ Hadassah Medical Center, Gynecologic Oncology, Jerusalem, Israel; ${ }^{2}$ Cheba Medical Center, Gynecologic Oncology, Ramat Gan, Israel; ${ }^{3}$ Rabin Medical Center, Gynecology, Petah Tikva, Israel; ${ }^{4}$ Assaf Haroffe Medical Center, Sackler School of Medicine, Gynecology, Zrifin, Israel; ${ }^{5}$ Carmel Medical Center, Obstetrics and Gynecology, Haifa, Israel; ${ }^{6}$ Kaplan Medical Center, Hebrew University, Gynecology, Rehovot, Israel; 'Rambam, Gymecologic Oncology, Haifa, Israel; ${ }^{8}$ Wolfson Medical Center, Holon, Sackler Faculty of Medicine, Tel Aviv University, Gynecology, Tel Aviv, Israel; ${ }^{9}$ Ahmet, Gynecology, Ashkelon, Israel; ${ }^{10} \mathrm{ZIV}$, Gynecologic Oncology, Zefat, Israel; ${ }^{11}$ Poriah, Gynecologic Oncology, Tiberia, Israel; ${ }^{12}$ Hillel Yafe, Gynecology, Hadera, Israel; ${ }^{13}$ Barzilai Medical Center, Gynecology, Ashkelon, Israel

10.1136/ijgc-2021-IGCS.201

Objectives To evaluate the association of lower uterine segment involvement (LUSI) in high-grade endometrial cancer (HGEC) with oncological outcome.

Methods We performed a retrospective multi-center cohort study of consecutive women with HGEC stages I-III who underwent surgery in nine gynecological oncology centers in Israel. Recurrence-free and overall survival were compared between both groups. Univariate, Kaplan-Meier survival and Cox proportional hazard model analyses were used.

Results Overall 432 women, 152 with and 280 without LUSI were followed for a median time of 35 months (interquartile range 17-71). Both groups were comparable in demographical and medical history characteristics. Cancer histological type did not differ between groups with uterine serous carcinomas and grade 3 composing $39.1 \%$ and $33.3 \%$ of the cohort. Carcinosarcoma and clear cell histology composed the rest. Women with LUSI had higher rates of $\geq$ stage II disease $(58.6 \%$ vs. $22.1 \%, \mathrm{p}<0.001)$ and lower rate of lymphovascular space invasion (LVSI) $(66.4 \%$ vs. $79.3 \%, \mathrm{p}=0.003)$. LUSI was associated with an Odds Ratio for disease recurrence of 1.7 (95\% Confidence Interval 1.1-2.6). Univariate survival analysis underlined shorter median overall survival among LUSI women (28 months vs. 41, p<0.001). Cox proportional hazards model adjusted for LVSI, age, disease stage and chemotherapy demonstrated that LUSI was not independently associated with decreased OS.

Conclusions In women with HGEC, the presence of LUSI is not an independent poor prognostic factor.

\section{EPV132/\#552 THE PROGNOSTIC IMPACT OF LOWER UTERINE SEGMENT INVOLVEMENT IN WOMEN WITH LOW- RISK ENDOMETRIAL CARCINOMA: A MULTICENTER STUDY}

${ }^{1} \mathrm{G}$ Levin*, ${ }^{2} \mathrm{~B}$ Brandt, ${ }^{3} \mathrm{~L}$ Helpman, ${ }^{4} \mathrm{R}$ Eitan, ${ }^{5} \mathrm{Z}$ Vaknin, ${ }^{6} \mathrm{O}$ Lavie, ${ }^{7} \mathrm{~A}$ Ben Arie, ${ }^{8} \mathrm{~A}$ Amit, ${ }^{9} \mathrm{~T}$ Levy, ${ }^{10} \mathrm{~A}$ Namazov, ${ }^{11} \mid \mathrm{I}$ Ben Shachar, ${ }^{12}$ Atlas, ${ }^{13} \mathrm{I}$ Bruchim, ${ }^{1} \mathrm{~T}$ Perri, ${ }^{14} \mathrm{O}$ Gemer. ${ }^{1}$ Hadassah Medical Center, Gynecologic Oncology, Jerusalem, Israel; ${ }^{2}$ Hadassah, Gynecologic Oncology, Jerusalem, Israel; ${ }^{3}$ Cheba Medical Center, Gynecologic Oncology, Ramat Gan, Israel; ${ }^{4}$ Rabin Medical Center, Gynecology, Petah Tikva, Israel; ${ }^{5}$ Assaf Haroffe Medical Center, Sackler School of Medicine, Gynecology, Zrifin, Israel; ${ }^{6}$ Carmel Medical Center, Obstetrics and Gynecology, Haifa, Israel; '72. Kaplan Medical Center, Hebrew University, Gynecology, Rehovot, Israel; ${ }^{8}$ Rambam, Gymecologic Oncology, Haifa, Israel; ${ }^{9} 8$ Wolfson Medical Center, Holon, Sackler Faculty of Medicine, Tel Aviv University, Gynecology, Tel Aviv, Israel; ${ }^{10}$ Ahmet, Gynecology, Ashkelon, Israel; ${ }^{11}$ ZIV, Gynecologic Oncology, Zefat, Israel; ${ }^{12}$ Poriah, Gynecologic Oncology, Tiberia, Israel; ${ }^{13} \mathrm{Hilllel}$ Yafe, Gynecology, Hadera, Israel; ${ }^{14}$ Barzilai Medical Center, Gynecology, Ashkelon, Israel

\subsection{6/ijgc-2021-IGCS.202}

Objectives To study whether lower uterine segment involvement (LUSI) correlates with oncological outcomes in women with stage IA endometrial carcinoma with low risk features.

Methods We performed a retrospective multi-center cohort study of consecutive women with stage IA EC, who underwent complete surgical staging in eight gynecologic oncology centers in Israel. We included only women with grade 1-2 endometrioid histology, with negative lymphovascular space invasion, and those who did not receive adjuvant therapy. Univariate analysis, Kaplan-Meier survival and Cox proportional hazard models analysis were used to compare survival outcomes between women with and without LUSI.

Results We identified 283 cases for analysis. LUSI was diagnosed in 25 (8.8\%). Media follow up was 72 months (interquartile range 40-144). There were no significant differences between both groups with regard to the following parameters: age, medical history, duration of symptoms, tumor grade and time from diagnosis to surgery. Overall 5year survival and 5-year progression free survival were similar between the groups ( $\log$ rank test $p=0.993, p=0.244$, respectively). Recurrence rate did not differ between groups $(0 \%$ in LUSI vs. $5.0 \%$ in No LUSI groups, $\mathrm{p}=0.614)$. In Cox regression model adjusting for age, comorbidities and tumor grade - LUSI was not associated with overall survival $(\mathrm{p}=0.556)$.

Conclusions In women with stage IA EC with low-risk features, the presence of LUSI does not correlate with oncological outcome. LUSI as a sole finding should probably not dictate a decision upon adjuvant management in this low-risk population. 